\title{
IMPACTO DE LA APLICACIÓN DEL ANTICIPO DE IMPUESTO A LA RENTA
}

\author{
IMPACT OF THE ADVANCE INCOME TAX APPLICATION \\ NÉStor Daniel GutiérRez Jaramillo* \\ Unidad de Posgrado de la Facultad de Ciencias Contables \\ Universidad Nacional Mayor de San Marcos - UNMSM / Lima - Perú \\ [Recepción: Noviembre de 2015/ Conformidad: Diciembre 2015]
}

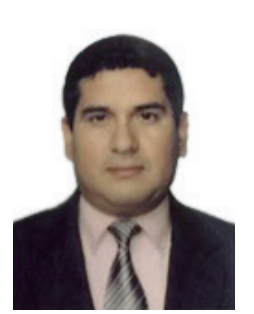

\section{RESUMEN}

El Anticipo Mínimo del Impuesto a la Renta en la República del Ecuador, es una de las cargas impositivas que mayor controversia genera entre los contribuyentes, debido a su naturaleza de impuesto heterodoxo que grava renta en función de cálculos sobre el patrimonio, activos, costos, gastos e ingresos que no guardan relación con la utilidad que se determina en el propio ejercicio fiscal. Esta carga impositiva forma parte de una medida encaminada a combatir la baja recaudación de impuestos directos originada por la evasión tributaria, que afecta directamente al Impuesto a la Renta. Para el desarrollo de la investigación, la información base se solicitó al Servicio de Rentas Internas mediante requerimiento, así como también, se utilizaron las bases de datos que constan en la página web de dicha institución pública, las que fueron procesadas de forma estadística. Además se aplicaron encuestas que permitieron conocer la percepción de los contribuyentes, sociedades de la provincia de El Oro. Como resultado se estableció que las reformas tributarias correspondientes al periodo 2006-2012 contribuyeron al incremento de la carga impositiva y a la recaudación de impuestos directos e indirectos por parte del Gobierno en la provincia de El Oro, contribuyendo a fortalecer la progresividad del sistema recaudatorio.

\section{Palabras clave:}

Anticipo de impuesto a la renta; carga impositiva; impuestos directos e indirectos; progresividad.

\begin{abstract}
The minimum advance income tax in the Republic of Ecuador is one of the tax burdens that generates more controversy among taxpayers, due to its heterodox nature that levies taxes based on calculations of capital, assets, costs, expenses and income unrelated to the income determined for the fiscal year itself. This tax burden is part of a measure to combat low collection of direct taxes caused by tax evasion, which directly affects the income tax. For the development of the research the basic information was requested from the Internal Revenue Service and the databases available on the webpage of this public institution, which were processed statistically. We also applied surveys allowing us to know the perception of corporate taxpayers in the province of El Oro. As a result it was established that for the period 2006-2012 tax reforms contributed to the increase of the tax burden and collection of Direct and Indirect Taxes by the Government in the Province of El Oro, helping to strengthen the progressivity of the tax collection system.
\end{abstract}

\section{Keywords:}

Advance income tax; taxation; direct and indirect taxes; progressiveness.

\footnotetext{
* Doctorando de Ciencias Contables y Empresariales - UNMSM. Magíster en Administración de Empresas: Docente titular de la Universidad Técnica de Machala, Ecuador, E-mail: nestorgutierrezj@gmail.com/ngutierrez@utmachala.edu.ec
} 


\section{INTRODUCCIÓN}

El presente trabajo de investigación se fundamenta en la necesidad de realizar un análisis acerca de la incidencia de los cambios normativos al anticipo del Impuesto a la Renta durante el periodo 2006 al 2012 en la recaudación impositiva de la provincia de El Oro.

\section{PLANTEAMIENTO DEL PROBLEMA}

\section{Situación problemática}

Ecuador históricamente ha sido un país agroexportador, siendo el boom bananero mantenido en las décadas de los años 50 y 60, el punto de partida de la consolidación del país como productor de materias primas para la exportación, época en la cual la recaudación de impuestos se basaba principalmente en el Impuesto a la Renta, a las Ventas, a la Ley de Timbres, a los Aranceles Aduaneros y; en especial, a la incorporación del Ad Valorem.

Durante la década del cincuenta existió una primacía de los impuestos indirectos sobre los directos, en una relación promedio del 65\% al 35\% (Paz y Miño, 2015).

En el Ecuador, una de las causas principales por las que se presenta una baja recaudación en impuestos directos es la evasión tributaria, cuestión que afecta directamente al Impuesto a la Renta, e implica que los contribuyentes preferían cancelar al fisco impuestos que pueden trasladarse a terceros como es el caso del IVA, o en su defecto cumplir la función de agente recaudador a través de las retenciones en la fuente tanto de IVA como de Renta, más no cancelar impuestos con base en sus utilidades. (Andino \& Parra, 2007).

Respecto al tema, Almeida, Carrasco, Oliva, \& Maldonado (2012) manifestaban que los ingresos públicos en el Ecuador sufrían de tres síntomas de injusticia: la primera, las preasignaciones e inflexibilidades de los ingresos que limitaban el diseño de política pública a través de la priorización y uso eficiente de los mismos y que beneficiaban a pocos sectores de la sociedad; en segundo lugar, los excedentes de las empresas públicas ingresaban mínimamente al Estado, lo cual restringía el financiamiento de las obras de infraestructura que requería el país para su desarrollo; y el tercero, el sistema tributario se sustentaba en impuestos regresivos (IVA), exoneraba al gran capital del impuesto a la renta y dejaba espacio para la evasión y elusión generalizadas.

Otro aspecto relevante a considerar es que durante el lapso de tiempo de los años 2000 al 2006, el Ecuador se caracterizó por ser un país cuya economía se basaba en un modelo neoliberal donde la intervención del gobierno se reducía a lo básico y donde primaba la libre movilidad de los factores de producción sin mayores regulaciones estatales, modelo económico que estableció un esquema de política tributaria en el país orientada a recaudar lo mínimo e indispensable, sin respetar principios de justicia y equidad social, orientado a favorecer un sistema primario-exportador que concentra la riqueza en pocas manos (Oliva \& Amoroso, 2015).

Según la Secretaría Nacional de Planificación y Desarrollo (SENPLADES, 2009) el gobierno del presidente Economista Rafael Correa Delgado, electo en el año 2007, plantea como proyecto desarrollar un esquema estructural de planificación de gobierno denominado el plan nacional del buen vivir, dentro de este plan se consolida plantear en una nueva Constitución, la necesidad de que el sistema impositivo ecuatoriano priorice la recaudación de impuestos directos y progresivos, lo que obligaría a que se busquen mecanismos técnicos y legales para poder consolidar la recaudación de estos impuestos.

En el año 2008, se consolida el planteamiento de gobierno a través de la vigencia de una nueva normativa tributaria llamada Ley para la Equidad Tributaria en el Ecuador, donde se establece la creación de nuevas cargas impositivas y de reformas al mecanismo recaudatorio en el caso del Impuesto a la Renta de Personas Naturales y Personas Jurídicas.

La nueva forma de recaudar el Impuesto a la Renta estuvo dada con la determinación de un rubro denominado Anticipo Mínimo del Impuesto a la Renta, que consiste en el cálculo anticipado del impuesto a la renta a través de cuotas de pago y cuyo cálculo se realiza de manera aritmética, en el que se consideran los activos totales, los costos y gastos, el 
patrimonio y los ingresos, de esta forma se asegura que el contribuyente por lo menos cancele un rubro mínimo de Impuesto a la Renta durante un ejercicio fiscal.

\section{Formulación del problema}

En la presente investigación, el problema ha sido identificado respecto al impacto que ha ocasionado la aplicación de la nueva forma de cálculo y pago del anticipo de Impuesto a la Renta, bajo la concepción de impuesto mínimo, en la provincia de El Oro.

\section{Justificación}

Esta forma de cálculo del Impuesto a la Renta se vuelve controversial debido a que no se toma en consideración los resultados económicos del año sobre los cuales se debe liquidar el Impuesto a la Renta, sino que, por el contrario debe realizar una selección del valor mayor entre el impuesto causado del propio ejercicio económico y el anticipo calculado del Impuesto a la Renta para ese mismo periodo impositivo, la salvedad radica en que dicho anticipo se calcula con información del ejercicio fiscal anteriory se aplica una fórmula matemática que involucra para su cálculo al patrimonio, los activos, los ingresos y los costos y gastos.

Un tema de discusión radica en el hecho de que estos nuevos recursos que se recaudan a través del anticipo pueden ser un mero espejismo, ya que no reflejan la situación real de la economía al gravar un impuesto con resultados de un ejercicio económico totalmente distinto.

Por el motivo antes citado, el anticipo es una de las herramientas de recaudación más polémicas porque no se basa en el impuesto causado del ejercicio fiscal, es un adelanto por una obligación tributaria futura, el cual causa al contribuyente un costo financiero que es irrecuperable y al Estado, la incertidumbre de un aparente esquema de recaudación de renta.

\section{Objetivo general}

Evaluar el impacto económico de la evolución de los cambios normativos relacionados con la forma de cálculo y pago del Anticipo de Impuesto a la Renta en la Carga Impositiva, recaudación de Impuestos Directos e Indirectos por parte del Gobierno y la progresividad recaudatoria en los sectores económicos de la provincia de El Oro durante el periodo 2006-2012.

\section{MARCO TEÓRICO}

\section{Normas y regulaciones aplicables}

En el Ecuador la norma rectora sobre las condiciones que debe cumplir el sistema tributario es la Constitución de la República del Ecuador, luego por orden de jerarquía de normas se tiene que la Ley Orgánica de Régimen Tributario Interno es la que regula las condiciones específicas que se dan en las principales cargas impositivas del país, esto es Impuesto a la Renta e Impuesto al Valor Agregado.

$Y$ es justamente en las regulaciones del Impuesto a la Renta, que se puede encontrar al elemento principal de estudio en la presente investigación, esto es el Anticipo de Impuesto a la Renta, donde se norma su forma de cálculo, liquidación, pago, exoneraciones que se pueden aplicar y que están permitidos por mandato legal. Sin embargo, es importante acotar que la Norma Legal Tributaria ha cambiado en lo referente a la forma de liquidación y pago del Anticipo del Impuesto a la Renta desde el año 2006 hasta el año 2012.

En todas las reformas realizadas, la norma directamente involucrada es la Ley de Régimen Tributario Interno y el artículo reformado es el 41 que regula lo relacionado al cálculo y pago del Anticipo Mínimo del Impuesto a la Renta, motivo por el cual es de trascendental importancia que se detalle todos los cambios normativos relacionados a esta carga impositiva, objeto del presente estudio.

\section{Fundamento metodológico}

La presente investigación responde a un diseño cuantitativo de tipo no experimental debido a que no se modifican en forma intencional las variables independientes para conocer sus efectos sobre otras variables, específicamente lo que se realiza es observar los componentes de estudio en su contexto natural para proceder a analizarlos. Dentro de la investigación no experimental, se aplica un diseño transaccional donde se recolectan datos en un determinado lapso de tiempo, para luego proceder a describir las varia- 
bles y analizar su incidencia e interrelación, por lo que el diseño final de investigación responde a un diseño transaccional descriptivo y correlacional (Hernández, Fernández, \& Baptista, 2010).

Además, se analiza la relación que existe entre la aplicación del Anticipo de Impuesto a la Renta y varias variables dependientes como el pago de Impuestos Directos e Indirectos, la Carga Impositiva y la Progresividad Recaudatoria de los sectores económicos de la provincia de El Oro durante el periodo 2006-2012.

Se realiza un análisis detallado de la relación existente entre las variables con el fin de que la investigación se utilice como fuente de reflexión a la comunidad Orense, respecto a la incidencia de la política tributaria a través de una carga impositiva específica, sobre la recaudación de Impuestos Directos e Indirectos, la progresividad recaudatoria y el desarrollo de los sectores económicos de la provincia de El Oro.

\section{ANÁLISIS, INTERPRETACIÓNY DISCUSIÓN DE RESULTADOS}

Los encuestados consideran que durante el periodo 2006-2012 los cambios normativos relacionados al anticipo del Impuesto a la Renta contribuyeron al incremento de la carga tributaria del sector económico donde se desempeñan, debido a que el 41,9\% y el 51,3\% respectivamente, respondieron de forma positiva y solamente el 6,7\% de forma negativa. La percepción de los encuestados guarda consistencia con los resultados de la recaudación de la provincia de El Oro durante los años 2006 al 2012, principalmente en el hecho de que al convertirse en un impuesto mínimo el anticipo del Impuesto a la Renta, se incrementó la recaudación por este concepto en relación al total recaudado (Gráfico oo 01).

\section{Gráfico No 01}

Percepción de los encuestados respecto al incremento de la carga tributaria del sector económico en el que se desempeña (\%)

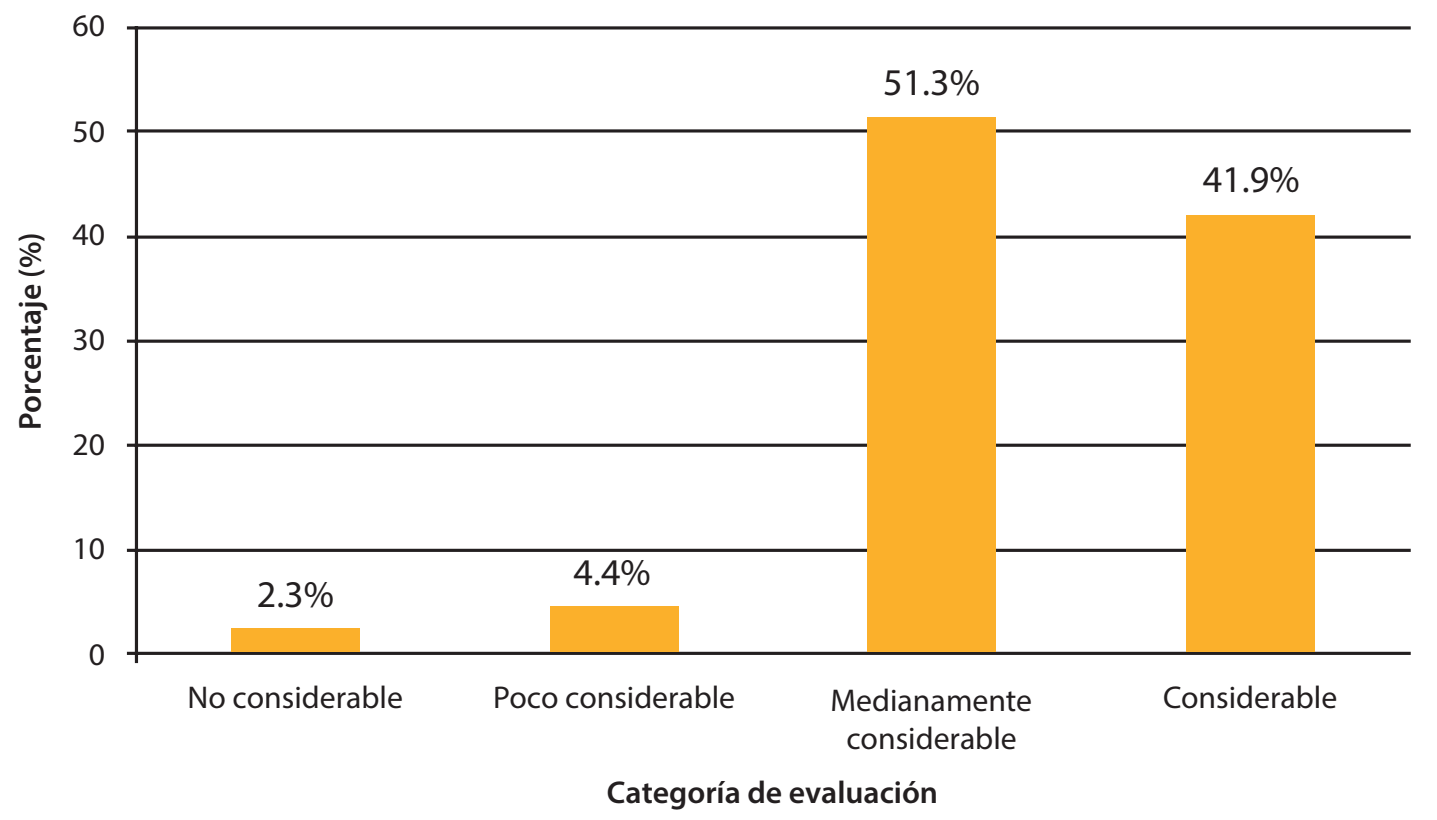

Fuente: Elaboración propia.

Para establecer la carga impositiva que ha generado el Anticipo Mínimo del Impuesto a la Renta en la provincia de $\mathrm{El}$ Oro, se procedió a considerar el impacto del anticipo calculado que declararon los contribuyentes sobre la recaudación total de impuestos en los distintos sectores económicos de la provincia, en los resultados se puede apreciar que en el año 2008 se incrementó a 8,74\% la relación, por motivo

22/ QVIPUKAMAYOC | Vol. 23(44) 2015 
de la aplicación del nuevo anticipo del Impuesto a la Renta. Este crecimiento continuó incluso en el año 2009 donde la relación alcanzó el 11,30\%, crecimiento considerable que guarda relación con el hecho de que durante estos años existió la posibilidad de que los contribuyentes consideren la compensación o devolución de los saldos a favor que se originen por el pago del anticipo de Impuesto a la Renta.
Sin embargo, desde el año 2010 al entrar en vigencia el anticipo de Impuesto a la Renta bajo la concepción de Impuesto Mínimo, se verifica que comienza una nueva tendencia al incremento de la relación desde el año 2010 hasta el 2013, lo que determina que los cambios normativos en referencia al Anticipo del Impuesto a la Renta incrementaron la carga fiscal por este concepto en los distintos sectores económicos de la provincia de El Oro (Tabla $\left.\mathrm{N}^{\circ} 01\right)$.

Tabla No 01

Anticipo calculado y Recaudación total de los sectores económicos en la provincia de El Oro durante el periodo 2006-2012

\begin{tabular}{|l|c|c|c|}
\hline Años & Anticipo calculado $(\mathbf{\$})$ & Recaudación total $(\mathbf{\$})$ & $\%$ \\
\hline 2007 & $1.002 .173,91$ & $41.371 .783,88$ & 2,42 \\
\hline 2008 & $5.245 .963,17$ & $60.010 .575,87$ & 8,74 \\
\hline 2009 & $6.991 .707,40$ & $61.884 .083,68$ & 11,30 \\
\hline 2010 & $7.864 .334,03$ & $77.900 .061,55$ & 10,10 \\
\hline 2011 & $10.194 .741,80$ & $93.378 .418,77$ & 10,92 \\
\hline 2012 & $12.464 .482,30$ & $117.368 .990,80$ & 10,62 \\
\hline 2013 & $15.194 .006,30$ & $128.899 .012,80$ & 11,79 \\
\hline
\end{tabular}

Fuente: Elaboración Propia.

En el Gráfico No 02, se aprecia como la tendencia de la Carga Impositiva del Anticipo del Impuesto a la Renta se incrementa por año, sobre todo desde la concepción del Anticipo como Impuesto Mínimo que entró en vigencia desde el año 2010.

\section{Gráfico No 02}

Porcentaje de Carga Impositiva del Anticipo del Impuesto a la Renta en el periodo 2007-2013 y predicción para los años 2014-2015

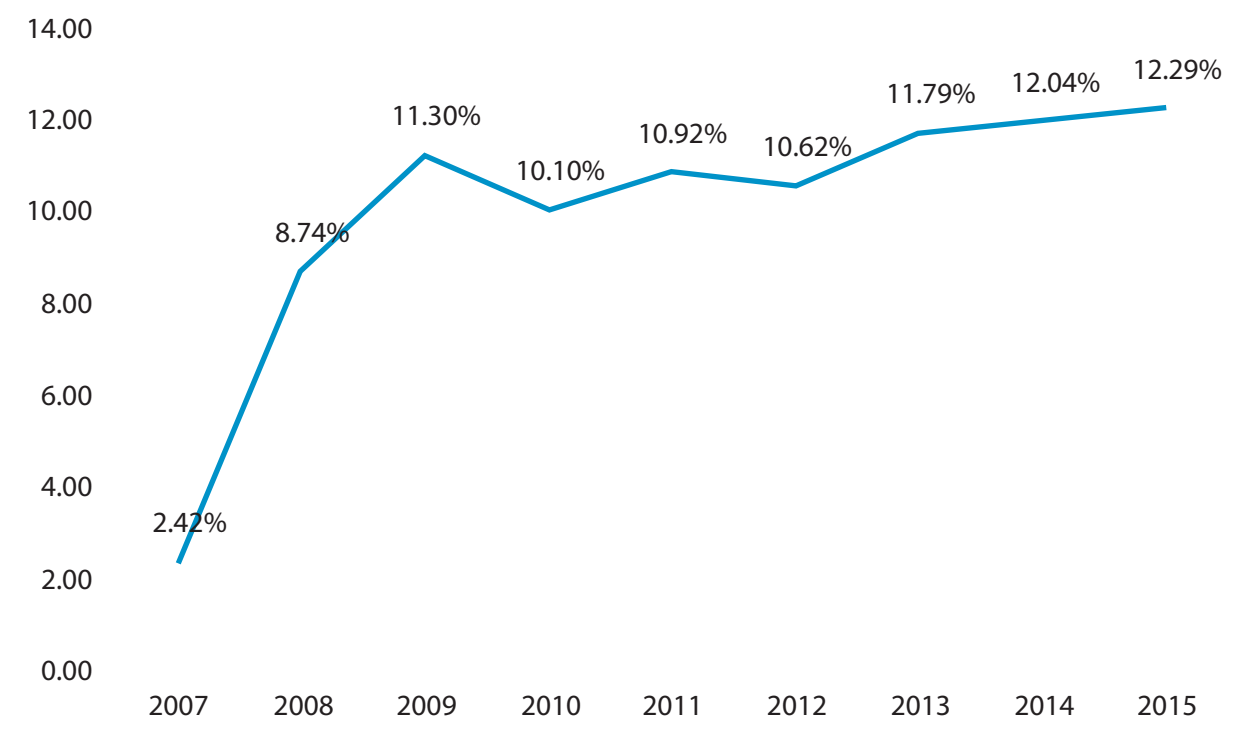

Fuente: Elaboración propia. 
En relación con el impacto económico del pago del Anticipo del Impuesto a la Renta en el flujo de efectivo de su empresa el $37,2 \%$ y el $57,0 \%$ de los encuestados manifiestan que ha sido considerable y medianamente considerable respectivamente y el $5,7 \%$ plantean que no ha presentado una influencia marcada. La percepción de los encuestados es que se presenta un relevante efecto sobre el flujo de efectivo de las empresas a partir de la aplicación del anticipo de Impuesto a la Renta, es importante recordar que al ser el Anticipo de Impuesto a la Renta un impuesto Mínimo que se cancelará por este concepto, es razonable que se afecte al flujo de efectivo de las empresas, toda vez que el anticipo no discrimina la situación económica real de las empresas, ya que pierda o gane en el ejercicio fiscal como mínimo cancelará por Impuesto a la Renta este concepto (Tabla $\mathrm{N}^{\circ} 02$ ).

Tabla No 02

Percepción de los encuestados en relación al impacto económico del pago del Anticipo de Impuesto a la Renta en el flujo de efectivo de la empresa

\begin{tabular}{|l|c|c|c|c|}
\hline \multicolumn{1}{|c|}{ Categorías de evaluación } & Frecuencia & Porcentaje & Porcentaje válido & Porcentaje acumulado \\
\hline No considerable & 4 & 1,3 & 1,3 & 1,3 \\
\hline Poco considerable & 13 & 4,4 & 4,4 & 5,7 \\
\hline Medianamente considerable & 170 & 57,0 & 57,0 & 62,8 \\
\hline Considerable & 111 & 37,2 & 37,2 & 100,0 \\
\hline \multicolumn{1}{|c|}{ Total } & $\mathbf{2 9 8}$ & $\mathbf{1 0 0 , 0}$ & $\mathbf{1 0 0 , 0}$ & \\
\hline
\end{tabular}

Fuente: Elaboración Propia.

Por otro lado, se tiene que la tendencia de la recaudación de Impuestos Directos e Indirectos por parte del Gobierno en relación a la Recaudación total denota la prevalencia de los primeros sobre los segundos, lo que se encuentra asociado a que los cambios normativos contribuyeron a priorizar la recaudación de impuestos directos y cumplir con los preceptos constitucionales, durante el periodo 2007 al 2013. (Gráfico No 03).

\section{Gráfico No 03}

Recaudación de Impuestos Directos e Indirectos por parte del Gobierno por sector económico de la provincia de El Oro (\$)

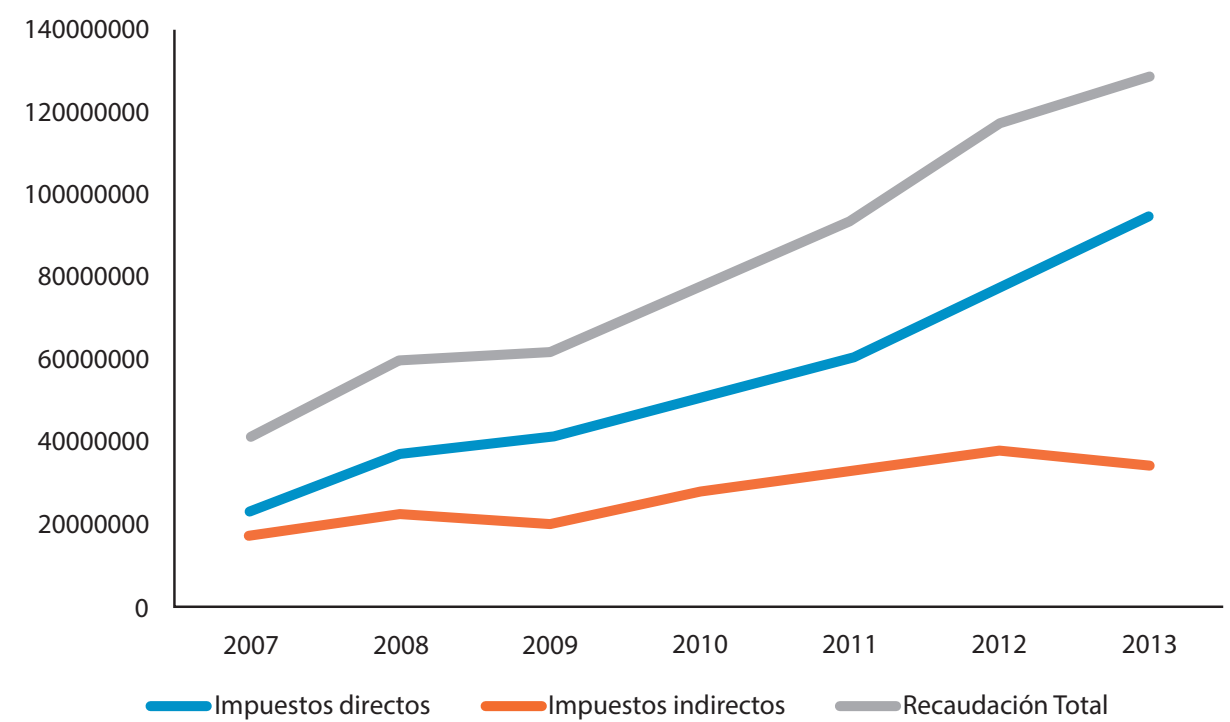

Fuente: Elaboración propia. 
En la provincia de El Oro a partir de las reformas tributarias ocurridas en el periodo 2006-2012, ha prevalecido la recaudación de Impuestos Directos por sobre los Indirectos. Con este hecho, es importante evaluar la tendencia general de recaudación en la pro- vincia de El Oro, donde se verifica que el sector que mayor contribuye a la recaudación es el de comercio al por mayor y menor, seguido por el de explotación de minas y canteras, pesca e intermediación financiera (Gráfico No 04).

\section{Gráfico No 04}

Recaudación total generada por los principales sectores económicos de El Oro en el periodo 2007-2013 (\$)

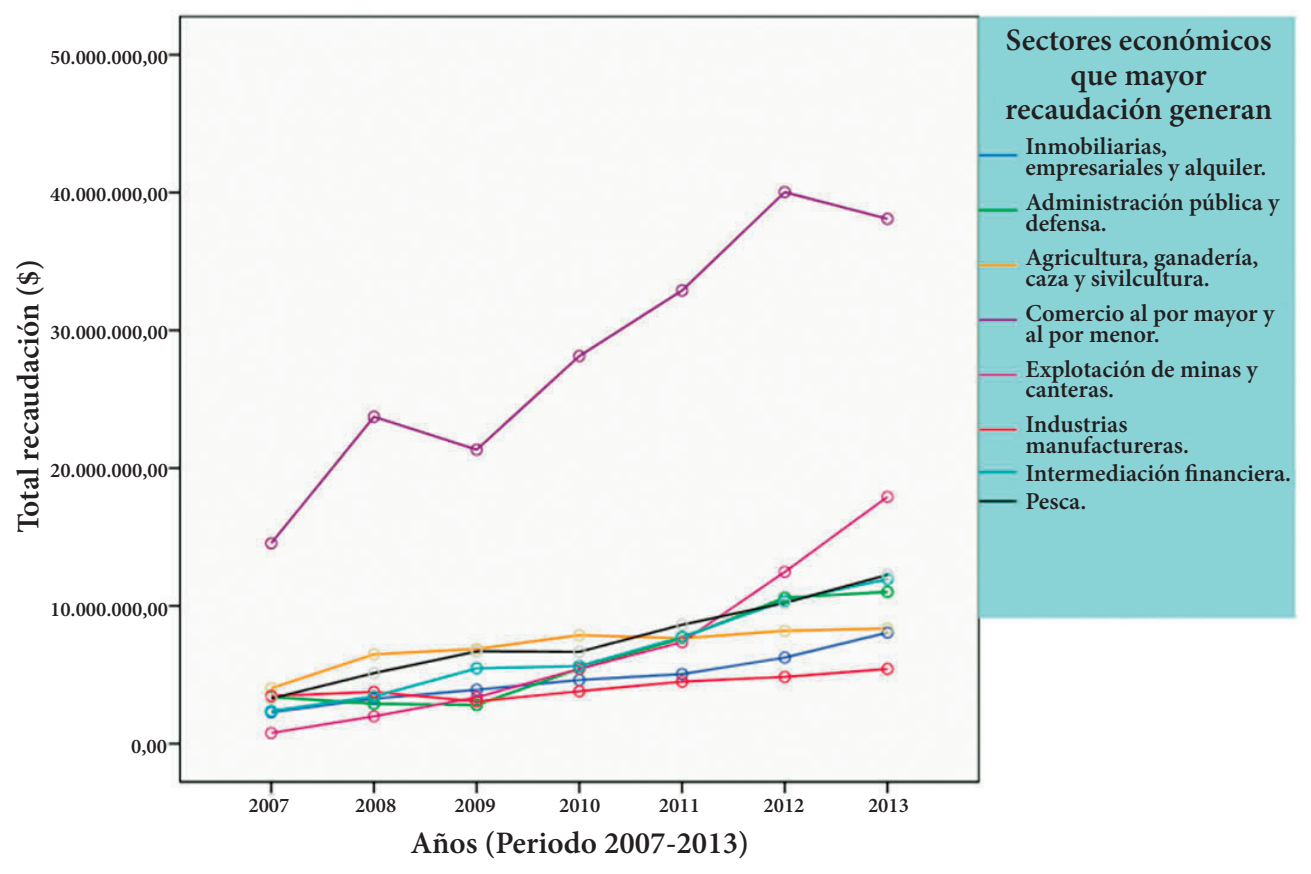

Fuente: Elaboración propia.

El incremento de la carga impositiva del Anticipo del Impuesto a la Renta junto con la prevalencia de recaudación de Impuestos Directos sobre los Indirectos, sumada a la tendencia de la recaudación del Impuesto a la Renta en la provincia de El Oro determinan el incremento de la progresividad en el sistema recaudatorio en el periodo 2007-2013. Lo que predice el modelo para cada año del periodo estudiado y para el año 2014, evidencian que los valores poseen una tendencia al incremento, por lo que se presupone que en el mismo se mantendrá la prevalencia de recaudación de Impuestos Directos sobre los Indirectos. (Gráfico No 03).

\section{Tabla No 03}

Serie cronológica que muestra los valores estimados de Impuesto a la Renta para el periodo comprendido entre 2007 y 2014 (\$)

\begin{tabular}{|c|c|}
\hline Años & Impuesto a la Renta(\$) \\
\hline 2007 & $41.371 .783,88$ \\
\hline 2008 & $60.010 .575,87$ \\
\hline 2009 & $61.884 .083,68$ \\
\hline 2010 & $77.900 .061,55$ \\
\hline 2011 & $93.378 .418,77$ \\
\hline 2012 & $117.368 .990,75$ \\
\hline 2013 & $128.899 .012,80$ \\
\hline 2014 & $141.372 .254,1$ \\
\hline
\end{tabular}

Fuente: Elaboración Propia. 


\section{CONCLUSIONES}

1. Desde la percepción de los contribuyentes el Anticipo del Impuesto a la Renta ha afectado de manera considerable a la rentabilidad y al flujo de efectivo de las empresas, sobre todo por su concepción de Impuesto Mínimo y por la falta de beneficios de exoneración y reducción cuando la actividad económica no genera renta alguna.

2. Las reformas tributarias realizadas durante el periodo 2006-2012 relacionadas con el pago del Anticipo del Impuesto a la Renta contribuyeron al incremento de la recaudación de Impuestos Directos e Indirectos por parte del Gobierno en la provincia de $\mathrm{El}$ Oro, predominando la recaudación directa sobre la indirecta.

3. En lo relacionado a la recaudación de Impuestos Directos se evidencia un crecimiento en los sectores económicos de la provincia de El Oro en el periodo 2006-2012, destacándose los sectores de Comercio al por mayor y menor, Explotación de minas y canteras, Pesca, Intermediación financiera, Agricultura, ganadería, caza y silvicultura.

4. Los cambios normativos ocurridos durante el periodo 2006-2012 incidieron en la creación de un sistema recaudatorio progresivo en la provincia de El Oro, debido a que se presenta una tendencia recaudatoria al alza en el periodo 2006-2013 y para los años 2014-2015.

\section{REFERENCIAS BIBLIOGRÁFICAS}

1. ALMEIDA, M., CARRASCO, C., OLIVA, N., \& MALDONADO, P. (2012). Ingresos y Gasto Público en democracia: de la rigidez fiscal al pago de la deuda social. En S. d. Internas, Una nueva Política Fiscal para el Buen Vivir (pág. 154). Quito, Ecuador: Abya-Yala.

2. ANDINO, M., \& PARRA, J. C. (2007). Estimación de la brecha de recaudación del IVA e Impuesto a la Renta de las sociedades por industria. Revista de Fiscalidad Internacional, pp 134-135.

3. OLIVA, N., \& AMOROSO, X. (2015). Nueva política tributaria para el cambio de época posneoliberal. En SRI, La Nueva Economía en la Nueva Constitución del Ecuador (pág. 102). Quito, Ecuador: Publingraf Industria Gráfica.

4. PAZ y MIÑO, J. (2015). Historia de los impuestos en Ecuador. Quito, Ecuador: (Digital Ed.) SRI-PUCE.

5. SENPLADES. (2009). Plan Nacional Para el Buen Vivir 2009-2013. Quito, Ecuador: El Conejo. 